\title{
Real Talk: Saturated Sites of Violence in CS Education
}

\author{
Yolanda A. Rankin* \\ Florida State University \\ Tallahassee, FL, USA
}

\author{
Jakita O. Thomas* \\ Auburn University \\ Auburn, AL, USA
}

\author{
Sheena Erete* \\ DePaul University \\ Chicago, IL, USA
}

\begin{abstract}
Despite numerous CS education pedagogical interventions, the pipeline of Black women in Computing has not increased, which illustrates the need to address structural issues (such as racism, sexism, power, and privilege) that impact Black women's intersectional identities. Without honest conversations about power relations within the field of Computing, one cannot expect to engender social change that equates to equity for all CS students. Leveraging intersectionality as a critical framework, we interview 18 Black women about their experiences navigating the computing education ecosystem. Intersectional analysis of Black women's experiences reveals that CS education consists of saturated sites of violence in which interconnected systems of power converge to enact oppression. Findings reveal three saturated sites of violence within CS education: 1 . traditional K-12 classrooms; 2. predominantly White institutions; and 3. internships as supplementary learning experiences.
\end{abstract}

\section{CCS CONCEPTS}

-Social and professional topics $\rightarrow$ Computing education programs.

\section{KEYWORDS}

Intersectional Computing; Black women; epistemic violence

\section{ACM Reference Format:}

Yolanda A. Rankin, Jakita O. Thomas, and Sheena Erete. 2021. Real Talk: Saturated Sites of Violence in CS Education. In Proceedings of the 52nd ACM Technical Symposium on Computer Science Education (SIGCSE '21), March 13-20, 2021, Virtual Event, USA. ACM, New York, NY, USA, 7 pages. https://doi.org/10.1145/3408877.3432432

\section{INTRODUCTION}

In 2020, we witnessed global civil unrest, protests, and uprisings as a result of the perpetual unfair treatment and murders of unarmed Black Americans by the police and White racists [1,28, 40]. Calls for social justice have emerged in every sector, including in Computing, demanding structural change to address historical inequities that exist due to the unfair treatment of Black people. Via letters to the ACM leadership, blog posts, and other public forms of communication, Black scholars have added their voices to the cries for social justice, sharing painful personal experiences that point to the

*All authors contributed equally and are recognized as first authors of this paper.

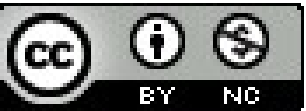

This work is licensed under a Creative Commons Attribution-NonCommercial International 4.0 License.

SIGCSE '21, March 13-20, 2021, Virtual Event, USA.

(C) 2021 Copyright held by the owner/author(s).

ACM ISBN 978-1-4503-8062-1/21/03.

https://doi.org/10.1145/3408877.3432432 existence of racism in the field of Computing [3, 15, 24, 35, 48]. To our astonishment, White allies and scholars, who have conducted decades of CS education research that supposedly pushes for inclusion and equity for minoritized populations, shared their lack of awareness of the racism and differential treatment that Black people experience on a daily basis [22].

CS education has endorsed the false narrative of being a colorblind discipline which, as an ideology, is racist, because it refuses to acknowledge race as a contributing factor to the differential treatment that racialized groups experience [5]. Colorblind ideology maintains Whiteness as the established norm, rendering people of color as being invisible in the field of Computing [5, 37]. Collins [8] refers to this kind of feigned ignorance as a camouflage for epistemic power in which those who are empowered and privileged within a particular interpretive community dictate the rules for what is considered acceptable knowledge. This concept of acceptable knowledge is deemed as that which reflects and protects the dominant group's status of power and privilege (epistemic oppression) while rejecting and silencing other forms of knowledge produced by marginalized populations (epistemic violence)[8].

Recent studies conducted by Black scholars point to examples of oppression and trauma that Black people, specifically Black women, have endured in order to persist in the field of Computing $[33,35,36,44,47]$. Findings from this research testify to the hostile culture of CS education and reveal that systemic oppression (racism, gendered racism, etc.) does indeed exist in the field of Computing and represents acts of violence. Collins [8] suggests that scholars treat such violence as a saturated site of power relations. These "saturated sites bundle together practices, social institutions, representations, and patterns of everyday social interaction that appear and reappear across seemingly separate systems of oppression. Saturated sites are important because their hypervisibility and ubiquity makes the points of convergence or transactions of intersecting power relations more visible" [8, p. 238]. We argue that these saturated sites of power relations represent violence because of the physical, emotional, mental, spiritual, professional, and academic damage that they inflict on Black women (as described in $[36,44])$, but also because of the damage they cause to the field of Computing (as described in [29]). It is a form of violence that does not leave visible wounds but harms and injures, nonetheless.

As an act of resistance, we take this opportunity to call out how power functions within Computing to perpetuate systemic discrimination and racism that negatively impact Black people, particularly, Black women, and other marginalized populations in the field of Computing. We begin this examination by identifying saturated sites of epistemic violence within CS education as described from the first-hand accounts of Black women in the field as they navigate spaces in secondary and post-secondary CS education. As such, we pose the following research question: What are the saturated sites of epistemic power in CS education for Black women? 
As a resistant knowledge project, we aim to disrupt the homogeneous treatment of "women of color" who are often lumped into one group to preserve statistical significance, when that categorization represents incredibly heterogeneous populations of Black, Latina, Native American, Asian Pacific Islander and other marginalized women who each deserve to be centered in their own right and not compared to other dominant groups [48]. In service to intersectional Computing [35, 44], we hope that this work and its methods will be taken up by members of other historically excluded groups within Computing and replicated so that we can begin to understand the commonalities and differences among marginalized populations across multiple axes (e.g., race, gender, class, nation, ability).

\section{THEORETICAL FRAMEWORK}

Within the context of CS education research, we intentionally center the experiences of Black women in Computing [36, 44], applying intersectionality as a critical framework for understanding power, privilege, and saturated sites of violence.

\subsection{Intersectional Computing}

Since the late 1990s, a burgeoning body of CS education research that emphasizes the recruitment and retention of women in Computing has emerged [4, 6, 18, 20,21, 26], drawing attention to how the "one size fits all" pedagogical approach caters more to men/boys while alienating women/girls. Though CS education has managed to pivot in an attempt to better cater to women [2, 18, 19, 38, 43], less research has addressed the issue of systemic gendered racism that women of color, in particular Black women, face in Computing $[36,42,44,49]$. Given Black women's unique vantage point of being both Black and women [7, 41], adequate analytical tools become necessary for examining the intersection of race and gender as interconnected systems of power that oppress women of color. As such, intersectionality, a critical social theory for examining the complexity of how "systems of power co-produce one another in ways that reproduce both unequal material outcomes and the distinctive social experiences that characterize people's experiences within social hierarchies," [8, p.46] has begun to gain momentum in the field of Computing.

Despite the uptake of intersectionality within Computing, we raise serious concerns about how our colleagues are misappropriating intersectionality, ignoring key aspects of what makes intersectionality a critical form of inquiry and praxis in the fight for social justice in the context of CS education [9]. One of the guiding premises of intersectionality is its ability to expose the inner workings of power relations that create social inequalities for marginalized populations [8]. Collins describes these power relations as four distinctive domains: 1 . structural - how power is organized; 2 . cultural - subliminal messages about which social groups are included and excluded; 3. interpersonal - how social interactions privilege one social group over another; and 4. disciplinary - how different sets of rules are applied arbitrarily to different social groups. These four domains represent a dominating matrix of intersecting power dynamics that exemplifies racism, sexism, socioeconomic status, homophobia, ableism, xenophobia, etc.

Intersectional research in the context of CS education has failed to address issues of power and privilege. Introducing the concept of saturated sites of violence as interconnected systems of power that converge in epistemic violence, Collins [8] describes the inner workings of power within and across oppressive systems, making them visible while providing "an entry point into theorizing intersecting systems of power" [8, p.238]. Detailed descriptions of sites of violence allows us to examine the "conceptual glue" that binds intersecting systems of power together (ibid). This is significant, because oppression is used to justify dominant narratives that seek to explain why things are the way they are. This opens up "new pathways for conceptualizing domination" used to subjugate, surveil, and enforce the assimilation of less dominant social groups. Finally, it "sheds light on resistant knowledge projects of anti-violence initiatives" (ibid). This includes strategies that Black women develop to cope, navigate, and fight even as they experience saturated sites of epistemic violence in the field of Computing.

For example, a dominant narrative that is prevalent in CS education at the undergraduate level is that students are most likely to succeed in Computer Science as a major if they have been exposed to Calculus in high school, have family and friends in the field, and have early exposure to CS and programming experience prior to college [39]. Students who enter undergraduate CS education differently from the dominant narrative often struggle, not because they are incapable of succeeding in Computing, have a weak computing identity or lack "grit", but rather because the dominant narrative views their differences as deficiencies and determines them to be less "fit" or not "CS material" [12, 44]. As a result, proper supports do not exist, as the assumption is that everyone conforms or assimilates to the dominant narrative, resulting in a self-fulfilling prophecy. As we will see in this paper, Black women often contend with these dominant narratives and have to figure out ways to combat, resist, and overcome the hostile environments that exist because of these narratives, rife with assumptions that often push Black women to the margins of Computing. Intersectional Computing aims to provide CS education with the necessary vocabulary and tools (including theories and methods that have yet to be developed) for examining how power plays out in the field of Computing so that it can be identified, understood, and, ultimately, redistributed to those who have been disenfranchised.

\subsection{Power \& Privilege in CS Education}

Very little CS education research that seeks to increase diversity, inclusion, and equity discusses or even mentions the ramifications of power and privilege, and how these ramifications produce the social inequalities evident in the field of Computing [39, 46]. Such overt omission of the acknowledgement of power and privilege in the field of Computing enables intersecting oppression to continue to thrive on multiple levels. For example, the lack of acknowledgement of power conveys the message that Computing is a colorblind meritocracy, a level playing field in which anyone can succeed if she or he works hard enough, an example of the cultural domain of power $[9,36]$. However, Black women students tell a different story, one in which they experience rejection from their non-Black classmates when seeking partners for group assignments (interpersonal power) or differential treatment (being ignored) compared to White women graduate students who receive mentorship regarding the unspoken rules for how to navigate graduate school [27]. 
Without an in-depth, honest conversation about power relations within the field of Computing, one cannot expect to engender social change that equates to equity, justice, and liberation in the field of Computing. Instead, the primary focus has been numerous studies of interventions that target underrepresented populations such as women, African Americans, LatinX, Native Americans, Asian Pacific Islanders and persons with disabilities [10, 11, 32, 34, 4547]. However, these same populations remain marginalized despite efforts to change the status quo $[16,50]$. This raises the question as to why have these interventions proven to be unsuccessful in significantly increasing the representation of historically excluded populations in CS? The answer to this question lies in the careful examination of power relations between dominant and non-dominant social groups in the field of Computing.

We call upon the intersectional experiences of Black women as legitimate "ways of knowing" [7, 33, 44] about how intersecting oppressions in CS education become saturated sites of epistemic violence. We examine Black women's experiences navigating the Computing ecosystem and identify CS education as a saturated site of violence. This represents a critical yet necessary departure from previous discussions about diversity, inclusion, and equity in CS education. We posit that racism, discrimination, sexism, abuse of power, dehumanization, etc. represent various forms of violence that take place in CS education on a daily basis.

\section{POSITIONALITY STATEMENT}

Applying standpoint theory [23] as the motivation for our research, we include a positionality statement indicative of the research team. The research team includes three Black feminist scholars who have strong interests in the disruption of the dominant sociocultural norms attributed to the field Computing. Together, we represent 24 years of experience in the academe, conducting research that illustrates the complex ways in which Black women's bodies move through the world and specifically, the Computing ecosystem. Moreover, we use the term "Black women" to refer to all women of the African diaspora. Motivated by the need for more equitable, inclusive, and diverse formal and informal learning environments in CS education, our lived intersectional experiences provide a posteriori knowledge about marginalization in the field of Computing. Finally, we bracket our various sets of assumptions by including all experiences shared by our participants, not rejecting those that diverge from our own.

\section{METHOD}

\subsection{Participants and Settings}

Using the snowball sampling method, we initially sent an email to 4 Black women who were in different stages in the Computing pipeline (i.e., graduate students, early career professionals, and department faculty) to participate in semi-structured interviews and to recommend other Black women to participate. We repeated this cycle until we ultimately recruited a total of 18 U.S. Black women 8 CS faculty members, 2 government employees, 5 industry professionals, and 3 graduate students.

\subsection{Data Collection \& Analysis}

After obtaining IRB consent for each participant, one researcher conducted, recorded, and transcribed semi-structured interviews, asking a series of questions about their STEM identity, CS education trajectory and experiences, and factors that shaped their ability to persist in Computing. Note: The expressed views in this study do not reflect the perspectives of all Black women in Computing as we are not a monolithic population.

We conducted qualitative analysis of the 18 interviews representing Black women in various stages of the Computing pipeline. First, we read through each interview, identifying potential sites of violence based upon stages in CS education (e.g., K-12, post-secondary, undergraduate education and graduate education), type of learning environment (e.g., classrooms, out-of-school programs, boot camps, study sessions, and internships) and type of institution (Historically Black College/University, Predominantly White Institution, government research lab and industry). Next, using invivo coding, we coded all 18 transcripts to identify, in their own words, multiple examples in which Black women were oppressed. Applying an intersectional lens, we discussed which domain(s) of power was at work in each example and which type of oppression was described.

\section{FINDINGS}

We describe the three (3) most prevalent saturated sites of epistemic violence that emerged from the data: 1 . traditional K-12 classroom environments; 2. Predominantly White Institutions; and 3. internships as supplementary learning experiences.

\subsection{K-12 Traditional Classroom Environments}

According to Google's Computer Science Learning: Closing the Gap Black Students Brief, $80 \%$ of students in the U.S. who learned CS did so in a classroom setting, demonstrating that offering K-12 CS courses have the greatest potential for equitable access to CS education [25]. For the majority of Black women in our study, few had an opportunity to take a CS class in high school before matriculating into college. One participant shared her experience taking her first "CS class" in high school:

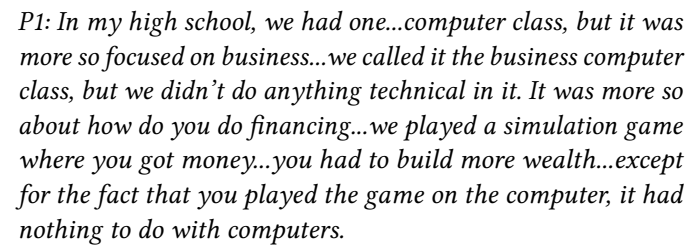

This particular Black woman describes a high school "business computer class" that has little to do with students learning core CS principles such as the distinction between software and hardware, algorithms, or programming. Though the class uses games to teach students about wealth management, students do not learn how to create video games. It is unknown if the teacher is even aware that the class does not satisfy the CS course curriculum for high school students. A significant antecedent, her high school is situated in a rural area and has limited resources (e.g. teachers who are not CS subject matter experts) to create a level playing field in which students are exposed and have access to computing as part of the high school curriculum. 
The structural domain of power becomes visible both through the lack of CS courses at this Black woman's high school and via the misrepresentation of course content as CS, when the course, in fact, has no relationship to CS education at all. The idea that labeling any high school class that leverages computers in the delivery of content as being related to or connected to computing, whether it actually is or not, results in a conceptual mismatch when those students reach college and realize that what they experienced in high school was not CS at all. This phenomenon is representative of a long tradition of unequal course offerings and resources in the U.S. when it comes to the education of Black children, one whose origins precede the Brown v. Board of Education decision in 1954 [31]. This is the "conceptual glue" that binds together the intersecting systems of racism, classism, and nation, in regards to who has what rights in this country, a battle that continues to this very day. This conceptual glue ensures that this Black woman enters her undergraduate CS program differently than the dominant narrative described earlier. Her lack of prior exposure serves to subjugate her as being "deficient," even though her path to computer science education at the undergraduate level was simply different.

In contrast, another participant in our study shares her learning experience in an introductory CS course at her high school:

\begin{abstract}
P2: In high school, my instructor, she was just not a very nice person...she wasn't very approachable...she's the type of instructor where if you ask her a question she kind of replies as if you're incompetent, so you don't want to ask her a question...she's going to make you feel stupid...she always kept the classroom cold...she would always keep her windows open even when it was wintertime and it was snowing outside...she said she wanted to minimize infections in her classroom...It's like she wanted us to hate coming to her class... she had her favorites, which of course I wasn't one.
\end{abstract}

In this scenario, this Black woman perceives her high school CS teacher, a White woman, as intentionally making students uncomfortable by subjecting them to chilly temperatures in the classroom. Power is revealed in the intersecting oppression of racism, classism, and ability (in terms of ability or "fitness" to engage in computing) as evident in the teacher's reaction to questions in the classroom. Instead of creating an environment where students' ideas, thoughts, and views are welcome, she engages in epistemic violence by refusing to answer their questions (silencing), which results in students censoring themselves (smothering) for fear of embarrassment and judgement. This now mature Black woman reflects on the situation, recognizing that the teacher used her authority via her social interactions with students (interpersonal domain of power) to create a hostile learning environment, one in which a young Black girl (at that time) becomes disadvantaged and oppressed. Her interest in learning CS is dampened and consequently, it is several years later before she realizes she enjoys programming, ultimately leading her to pursue an advanced degree in Computing.

These scenarios shed light on how high school CS classrooms can become saturated sites of violence for Black girls due to a lack of equitable resources (e.g., CS-enabled teachers, CS curriculum). Furthermore, White teachers who irresponsibly wield power are unaware that Black girls live and move differently through the world than White students and create inequitable and unjust learning environments that penalize Black girls at the high school level.

\subsection{Predominantly White Institutions}

Black women are vastly underrepresented in CS departments at Predominantly White Institutions (PWIs) [50]. Some of our participants represent alumnae of both PWIs and historically Black colleges/universities (HBCUs), receiving a mix of undergraduate and graduate degrees in Computing related fields. However, every Black woman who attended PWIs repeatedly describe hostile experiences at both the undergraduate and graduate level, making it clear that Black women are typically not welcome in computing departments at PWIs. One Black woman shares her experience as an undergraduate student at a reputable PWI:

P3: When I go there I really didn't know what to expect... just finding the group of friends that I could do homework with, study with, and all of that kind of stuff... a lot of the times people were very selective and didn't really want to include you in their studying...the Asian students wanted to study with people who were other Asians or the Indian students wanted to study with the students who are Indian.

This Black woman describes how racialized groups of students (i.e., Asians) study together, because there is a critical mass of them but too few Black students in the degree program to form their own study group. Though this experience takes place at a PWI, different racial groups are able to organize and support one another. However, these same groups do not welcome Black students as study partners. The underrepresentation of Black students insidiously implies that Black students are less capable of doing computing. Other racial groups judge Black students as being academically incompetent and inferior rather than their equals. Here, we see a specific type of discrimination in which certain racial groups, in this case Asian students, exclude Black students from their respective study groups. This is significant in that Asian students represent the second most dominant racial group in the field of Computing and arguably enjoy a certain degree of privilege denied to Black students [50]. This example testifies to the fallacy of colorblind pedagogical strategies, which fail to acknowledge or even address racial tensions between students of color, let alone the racial tension that exists between Black and White people. Because of "positive" stereotypes (e.g., smart, hard working) and larger numbers of Asian faculty and students in CS departments, Asian students may experience a certain degree of privilege.

Another Black woman shares her experience working with a group of three White males in a $\mathrm{C}++$ programming class:

P4: So it was this one project...It just so happened I was with three other boys and we were working on the project and we couldn't get it to work and I suggested approaching it in a different way and they were like, 'No, no, no, no, no, no.' I was like, 'We can keep doing what you're doing, but I'm pretty sure this is not going to work.' I wanted to be a team player...it was getting closer and closer to the deadline...I...recreated our project and did it all by myself... when I got it to work, I brought it back to the next team meeting we had. They were still struggling, trying to get their thing to work...I showed them what I did...they were like 'Oh my gosh, this is so great... Why didn't we think of this the first time?'...And I'm like yeah, well, I tried to say we should take it this way...they were still like 'Oh...yeah, this is kind of like what I said that one time.' And I'm like, 'No, it's not (laughs)...that whole experience was really, really frustrating for me. 
This scenario represents a common conundrum that Black women in Computing encounter on a regular basis. Her White male peers do not value her as an equal contributor in this group assignment and dismiss her initial attempts to engage in collaborative problem solving. Despite her efforts to be a team player, she is forced to work in isolation, and subsequently, experiences microagressions, where her idea was ignored, despite the fact that it was a working solution and her male colleague taking credit for the idea. Black women constantly deal with these kinds of microaggressions and cannot discern if it is their gender or race that causes their White male peers to dismiss them as being competent computer scientists.

In another conversation, one Black woman talks about the difficulty in finding research opportunities due to exclusion:

\begin{abstract}
P5: "I wasn't really kissing up to the right people...even though I had been doing research...since I got there.[..] I was seeing other people getting invited into these labs, and I wasn't.that kind of made me feel like well, maybe people just don't want me to work with them. Maybe they don't think I'm..smart enough... I just thought, okay, if I take your class, if I do well, you know, if I'm telling you that I'm interested and I'm talking [to] you, that that will get me an invite, and that just wasn't so.
\end{abstract}

This student realizes that graduate school works very differently from her undergraduate institution (an HBCU). During her first attempt to complete a Master's degree, she learns that it is customary to "kiss up" to a research advisor in order to receive an invitation to be a research assistant rather than solely based on one's academic performance, work ethic, or research potential. Despite her efforts, no one extends an invitation to her and she eventually discontinues the graduate program. The cultural domain of power sends the message that "Black women are not welcome here," creating an environment in which Black women are not valued but instead discouraged from completing advanced degrees despite interests in doing research. She questions her level of intelligence and her ability to do graduate level work since there is no guidance or support for understanding expectations and scholarly practices necessary for successfully obtaining a Master's degree.

One Black woman describes her sense of not belonging:

P6: They really don't know any better...I mean at the institution itself...getting those funny stares, people looking at you funny, people treating you like you're different, and you start to feel like you're different. And if you're not careful you'll think less of yourself or you'll be very, very shy. "

This particular Black woman talks about feelings of inadequacy, not being as competent or capable as her non-Black peers. Being hypervisible in a predominantly White landscape creates a level of self-consciousness that can negatively impact one's self-confidence. In this scenario, power is held by those outside of the Black community who stare at her, look at her funny, and treat her differently. She has no idea if this is happening because of her race, gender, or perceived class. These examples testify to the challenges that Black women face trying to "fit in" at PWIs.

\subsection{Internships as Learning Experiences}

While the traditional classroom environment largely contributes to CS education, internships supplement classroom instruction, providing opportunities for students to gain real world experience applying computing skills to solve real world problems. Unsurprisingly, students covet internships, because they acquire hands-on training and develop new skills that can be included on their resumes, giving them a competitive edge in the job market. Excited about traveling to a different geographical location known for its abundance of tech companies, this particular Black woman describes her summer internship experience:

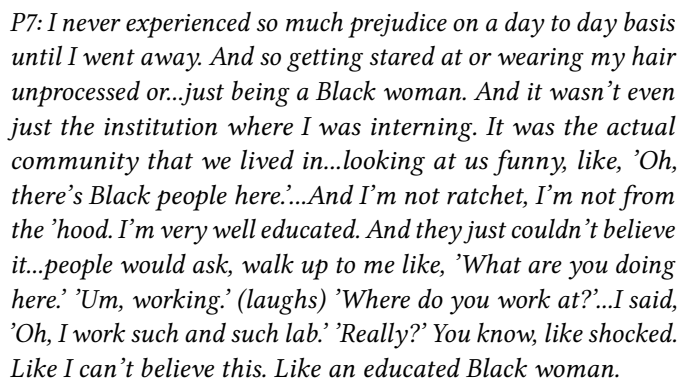
unprocessed or...just being a Black woman. And it wasn't even just the institution where I was interning. It was the actual community that we lived in...looking at us funny, like, 'Oh, there's Black people here.....And I'm not ratchet, I'm not from the 'hood. I'm very well educated. And they just couldn't believe it...people would ask, walk up to me like, 'What are you doing here.' 'Um, working.' (laughs) 'Where do you work at?'... I said, 'Oh, I work such and such lab.' 'Really?' You know, like shocked. Like I can't believe this. Like an educated Black woman.

This saturated site of epistemic violence involves the workplace, in what is designed to be an environment where students, as interns, can gain valuable work experience, understand and apply the concepts and content they are learning in CS classes to the "real world", and build their personal and professional networks by interacting not only with full-time employees of the company, but also with the cohort of other interns working for the company. This particular Black woman is disappointed to find this particular diverse geographical location to be hostile to Black women since people of different races, nationalities, and ethnicities live there. The internship experience becomes a saturated site of violence as this particular Black woman interacts with people who are residents of the city where her internship is located. The residents hold power, because of their knowledge of the city and understanding of its sociocultural norms. The workings of power across the oppressive systems of racism, sexism, and perhaps perceived classism are made visible in the way she is spoken to, the comments people make about her natural hair, and the looks she receives as she navigates the city and interacts with its residents. The residents' behavior implies that Black women, regardless of educational background or socioeconomic status, are an anomaly and do not belong in this professional laboratory or predominantly White community. This is evidenced by the surprise that the residents express once they learn that she is working for this company, suggesting that she is neither qualified nor educated enough to work for said company. This low expectation when it is discovered that they are educated or work in environments where employees possess specialized expertise is a common phenomenon that Black women experience in Computing and tech $[17,44]$. One Black woman succinctly states:

P8: You got to get thick skin because you're going to have to ... bypass any aggressions, whether they be micro or macro ...they're coming and they're not going to go away...if you can't focus yourself and you get easily distracted by those kinds of things, you're done...You're going to be in a lot of spaces where it's...uncomfortable. You're going to be alone...going to be marginalized...going to be considered less than...if you don't have good coping strategies, then it's going to be really hard. The sooner you develop those coping strategies, the better." 


\section{DISCUSSION}

This paper examines saturated sites of epistemic power and violence in CS education. Leveraging Black women's lived intersectional experiences, [7], our analysis reveals several insights.

Epistemic violence in CS education is constant for Black women and can occur at every level, academically, personally, and professionally. At the high school level, Black girls are relegated to the margins where they are expected to suffer in silence and not exercise their agency to ask questions or satisfy their intellectual curiosity. The attack continues on the campuses of PWIs where Black women in undergraduate and graduate CS classrooms become both invisible and hypervisible while continuously being pressured to assimilate into the dominant culture. It exists in interactions with classmates and faculty inside and outside the classroom as well as in research labs. It exists in the spaces where Black women live during their internship experiences while working for tech companies. What is clear from the testimonies of Black women is that saturated sites of violence are EVERYWHERE in CS education and Black women are constantly under seige without respite, except, perhaps among family and community $[33,44]$. It is no wonder we see the recent uprising, largely led by Black women, within the field of Computing, [14, 24, 30, 35, 36, 44].

As Black scholars in the field of Computing, we call for the CS education community to build coalitions with Black, Latina, Native American, and Asian Pacific Islander scholars who do this work within their communities. This creates a pathway to understand the particular "power grid" or matrix of intersecting power dynamics that affect each of these groups. Such research will dispel this notion of women of color in Computing as being homogeneous or having the same challenges. This suggests that, within CS education, we need to develop new theories, methods, and approaches to engage, understand, and support marginalized groups as they contend with saturated sites of epistemic violence.

Examining the power dynamics or conceptual glue uncovers assumptions about how students enter and navigate the Computing pipeline [8]. We posit that this conceptual glue consists of the assumptions and narratives that have been constructed and told by White people to define a wall of whiteness in Computing [37]. These assumptions and narratives are also used to tell stories (even if untrue) about why things in CS education are the way that they are. They are the mechanisms through which epistemic violence is justified and, "as a constellation of practices, violence is also essential to organizing and managing power as domination" [8, $\mathrm{p}$. 238]. Collins theorizes that "the routinized and normalized nature of violence, and the mechanisms that legitimize this entire endeavor from within distinctive systems of power become more visible within frameworks that see violence as one node of the connective tissue of systems of power" (ibid). Our intersectional analyses make salient the connective tissue of systems of power in CS education that Black women have experienced and described in their own words. A call to action to establish coalitions with Black women and remain in community with us is a prerequisite for discovering and understanding additional saturated sites of epistemic violence within Computing.

CS education is not a colorblind meritocracy. Rather, it is a matrix of intersecting oppressions, an interconnected system, for Black women in Computing. The concept of a system is a foundational concept in computer science. The way systems form, how they function, their outcomes, economies, components, and other aspects of systems and systems thinking undergird the field. We must apply that same understanding and rigor to analyzing how this dominating matrix of oppression marginalizes certain social groups in Computing to dismantle the systems and structures that have yielded the saturated sites of violence in CS education described in this paper and for those sites yet to be discovered. This paper serves as an initial step towards exposing the oppressive power structures embedded in CS education. Taking an interpersonal and systemic approach to transformation, we charge the community to engage in structural change to create a more equitable and just field of Computing.

\section{CONCLUSION \& FUTURE WORK}

Future work requires the intentional deconstruction of oppressive power structures, starting with K-16 CS education that leverages Black people, our history, and our culture as assets and resources for the benefit of all students. For those who wield power and benefit from these systems of oppression, they must do the work of demolishing them. If historically excluded groups understood how these oppressive structures were originally constructed, we would have dismantled them long ago. We did not build these systems and should not be responsible for destroying them. Furthermore, an emphasis on policy alone (requiring K-12 students to take CS courses) will not address the systems of intersecting oppression if educators who teach Black students bring deficit-model thinking and other biases into the classroom, subsequently, treating Black students disparagingly[13].

We argue that future research that identifies additional sites of violence in the field of Computing and how epistemic violence operates is necessary for three reasons. First, we need to hold the field of Computing accountable for its role in enabling oppression, which has resulted in the exclusion of Black people. To hold the field accountable, we must expose how interlocking systems of power enable oppression in the field so that we know what it looks like and subsequently, can assess its impact. Second, Black women in Computing need more allies. Black women's lived intersectional experiences serve as evidence of how power and privilege operate in the field of Computing. To build progressive coalitions, we need more people to empathize with the collective standpoint of Black women in Computing and work with us to achieve equity [23, 44]. Finally, if we are truly committed to transforming CS education into an inclusive, diverse, and equitable community for all, then we must take steps to dismantle systemic oppression and its many manifestations in the field of Computing.

\section{ACKNOWLEDGMENTS}

This research is generously supported by the National Science Foundation Grant \#1937759.

\section{REFERENCES}

[1] Rachel Aretakis and Grace Hauck. 2020. Shooting death of Ahmaud Arbery in Georgia: What we know about the video, grand jury and arrests. https://www.usatoday.com/story/news/nation/2020/05/06/ahmaudarbery-jogger-shooting-what-know-video-grand-jury-arrests/5174327002/ 
[2] Jon Beck. 2007. Forming a Women's Computer Science Support Group. In Proceedings of the 38th SIGCSE Technical Symposium on Computer Science Education (Covington, Kentucky, USA) (SIGCSE '07). Association for Computing Machinery, New York, NY, USA, 400-404. https://doi.org/10.1145/1227310.1227451

[3] Quincy Brown. 2020. "On Being Black In Computing During These Days". https://medium.com/@quincykbrown/on-being-black-in-computingduring-these-days-54e049d56987

[4] Tracy Camp. 1997. The Incredible Shrinking Pipeline. Commun. ACM 40, 10 (Oct. 1997), 103-110. https://doi.org/10.1145/262793.262813

[5] Devon W. Carbado. 2013. Colorblind Intersectionality. Signs: Journal of Women in Culture and Society 38, 4 (2013), 13-19.

[6] J. McGrath Cohoon. 2002. Recruiting and Retaining Women in Undergraduate Computing Majors. SIGCSE Bull. 34, 2 (June 2002), 48-52. https://doi.org/10. 1145/543812.543829

[7] Patricia Hill Collins. 2000. Black feminist thought. Routledge.

[8] Patricia Hill Collins. 2019. Intersectionality as Critical Social Theory. Duke University Press.

[9] Patricia Hill Collins and Sirma Bilge. 2016. Intersectionality. Polity Press.

[10] Teresa Dahlberg, Tiffany Barnes, Kim Buch, and Audrey Rorrer. 2011. The STARS Alliance: Viable Strategies for Broadening Participation in Computing. ACM Trans. Comput. Educ. 11, 3, Article 18 (Oct. 2011), 25 pages. https://doi.org/10. 1145/2037276.2037282

[11] Jill Denner, Linda Werner, Jacob Martinez, and Steve Bean. 2012. COMPUTING GOALS, VALUES, AND EXPECTATIONS: RESULTS FROM AN AFTER-SCHOOL PROGRAM FOR GIRLS. Journal of Women and Minorities in Science and Engineering 18, 3 (2012), 199-213.

[12] Angela Duckworth and Angela Duckworth. 2016. Grit: The power of passion and perseverance. Vol. 234. Scribner New York, NY.

[13] Tisha Lewis Ellison. 2017. The matter in parents' stories: African American urban mothers' counter stories about the Common Core State Standards and quality teaching. Urban Education (2017), 1-31.

[14] Sheena Erete, Aarti Israni, and Tawanna Dillahunt. 2018. An Intersectional Approach to Designing in the Margins. Interactions 25, 3 (2018), 66-69.

[15] Sheena Erete, Yolanda Rankin, and Jakita Thomas. 2021. "I Can't Breathe: Reflections from Black Women in CSCW and HCI". Journal of the ACM HumanComputer Interaction CSCW (2021).

[16] National Science Foundation. National Center for Science and Engineering Statistics. 2019. Women, Minorities, and Persons with Disabilities in Science and Engineering: 2019. Special Report NSF 19-304. https://www.nsf.gov/statistics/wmpd Accessed on 2020-08-26.

[17] Kapor Center for Social Impact. 2017. Tech Leavers Study: A first-of-its-kind analysis of why people voluntarily left jobs in tech. http://www.kaporcenter org/wp-content/uploads/2017/08/TechLeavers2017.pdf

[18] Carol Frieze, Orit Hazzan, Lenore Blum, and M. Bernardine Dias. 2006. Culture and Environment as Determinants of Women's Participation in Computing Revealing the "Women-CS Fit". SIGCSE Bull. 38, 1 (March 2006), 22-26. https: //doi.org/10.1145/1124706.1121351

[19] Paula Gabbert and Paige H. Meeker. 2002. Support Communities for Women in Computing. SIGCSE Bull. 34, 2 (June 2002), 62-65. https://doi.org/10.1145/ 543812.543832

[20] Vashti Galpin. 2002. Women in Computing around the World. SIGCSE Bull. 34, 2 (June 2002), 94-100. https://doi.org/10.1145/543812.543839

[21] Denise Gürer and Tracy Camp. 2002. An ACM-W Literature Review on Women in Computing. SIGCSE Bull. 34, 2 (June 2002), 121-127. https://doi.org/10.1145/ 543812.543844

[22] Mark Guzdial. [n.d.]. "CS Teachers, It's (Past) Time To Learn About Race". https://cacm.acm.org/blogs/blog-cacm/245408-cs-teachers-its-past-timeto-learn-about-race/fulltext\#comments

[23] Sandra G. Harding. 2004. The Feminist Standpoint Theory Reader. (2004).

[24] Christina Harrington, Yolanda Rankin, Jasmine Jones, Robin Brewer, Sheena Erete, Tawanna Dillahunt, and Quincy Brown. 2020. A CALL TO ACTION FOR THE ACM. http://interactions.acm.org/blog/view/a-call-to-action-for-the-acm

[25] Google Inc. Gallup Inc. 2016. Computer Science Learning: Closing the Gap: Black Students. Technical Report. Findings from: Diversity Gaps in Computer Science Exploring the Underrepresentation of Girls, Blacks and Hispanics (Issue Brief No. 2). Retrieved from https://goo.gl/FIhXKp.

[26] Jane Margolis and Allan Fisher. 2002. Unlocking the clubhouse: Women in computing. MIT press.

[27] Terrell R. Morton and Eileen C. Parsons. 2018. \#BlackGirlMagic: The identity conceptualization of Black women in undergraduate STEM education. Science Education 102, 6 (2018), 1363-1393. https://doi.org/10.1002/sce.21477 arXiv:https://onlinelibrary.wiley.com/doi/pdf/10.1002/sce.21477

[28] Esme Murphy. 2020. 'I Can't Breathe!': Video Of Fatal Arrest Shows Minneapolis Officer Kneeling On George Floyd's Neck For Several Minutes. https://minnesota.cbslocal.com/2020/05/26/george-floyd-man-dies-afterbeing-arrested-by-minneapolis-police-fbi-called-to-investigate/

[29] Safiya Umoja Noble. 2018. Algorithms of oppression: How Search Engines Reinforce Racism. NYU Press.
[30] Ihudiya Finda Ogbonnaya-Ogburu, Angela D.R. Smith, Alexandra To, and Kentaro Toyama. 2020. Critical Race Theory for HCI. (2020), 1-16. https://doi.org/10. $1145 / 3313831.3376392$

[31] Charles J Ogletree. 2004. All deliberate speed: Reflections on the first half century of Brown v. Board of Education. WW Norton \& Company.

[32] Nichole Pinkard, Sheena Erete, Caitlin K. Martin, and Maxine McKinney de Royston. 2017. Digital Youth Divas: Exploring Narrative-Driven Curriculum to Spark Middle School Girls' Interest in Computational Activities. Fournal of the Learning Sciences 26, 3 (2017), 477-516. https://doi.org/10.1080/10508406.2017. 1307199

[33] Yolanda Rankin, Maedeh Agharazidermani, and Jakita Thomas. 2020. The Role of Familial Influences in African American Women's Persistence in Computing. In 2020 Research on Equity and Sustained Participation in Engineering, Computing, and Technology (RESPECT). 1-8.

[34] Yolanda Rankin and India Irish. 2020. A Seat at the Table: Black Feminist Thought as a Critical Framework for Inclusive Game Design. Proceedings of the ACM on Human-Computer Interaction (2020).

[35] Yolanda A. Rankin and Jakita O. Thomas. 2019. Straighten up and Fly Right: Rethinking Intersectionality in HCI Research. Interactions 26, 6 (Oct. 2019), 64-68. https://doi.org/10.1145/3363033

[36] Yolanda A. Rankin and Jakita O. Thomas. 2020. The Intersectional Experiences of Black Women in Computing. In Proceedings of the 51st ACM Technical Symposium on Computer Science Education (Portland, OR, USA) (SIGCSE '20). Association for Computing Machinery, New York, NY, USA, 199-205. https://doi.org/10.1145/ 3328778.3366873

[37] Sanjay Reddy. 2020. "On Performative Academic Anti-Racism". https: //reddytoread.com/2020/06/02/on-performative-academic-anti-racism/

[38] Eric S. Roberts, Marina Kassianidou, and Lilly Irani. 2002. Encouraging Women in Computer Science. SIGCSE Bull. 34, 2 (June 2002), 84-88. https://doi.org/10. $1145 / 543812.543837$

[39] Monique Ross, Zahra Hazari, Gerhard Sonnert, and Philip Sadler. 2020. The Intersection of Being Black and Being a Woman: Examining the Effect of Social Computing Relationships on Computer Science Career Choice. ACM Trans. Comput. Educ. 20, 2, Article 9 (Feb. 2020), 15 pages. https://doi.org/10.1145/ 3377426

[40] Jason Schreiber. 2020. "Students won't face charges for video mocking George Floyd death". https://www.unionleader.com/news/social issues/students-wontface-charges-for-video-mocking-george-floyd-death/article_9f11a7a4-d7dd5d42-8124-50c2771c51e3.html

[41] A. Solomon, D. Moon, A. L. Roberts, and J. E. Gilbert. 2018. Not Just Black and Not Just a Woman: Black Women Belonging in Computing. In 2018 Research on Equity and Sustained Participation in Engineering, Computing, and Technology (RESPECT). 1-5.

[42] Valerie E. Taylor. 2002. Women of Color in Computing. SIGCSE Bull. 34, 2 (June 2002), 22-23. https://doi.org/10.1145/543812.543821

[43] Joy Teague. 2002. Women in Computing: What Brings Them to It, What Keeps Them in It? SIGCSE Bull. 34, 2 (June 2002), 147-158. https://doi.org/10.1145/ 543812.543849

[44] Jakita O. Thomas, Nicole Joseph, Arian Williams, Chanrtel Crum, and Jamika Burge. 2018. Speaking Truth to Power: Exploring the Intersectional Experiences of Black Women in Computing. In Proceedings of the 2018 Research on Equity and Sustained Participation in Engineering, Computing, and Technology, RESPECT 2018. https://doi.org/10.1109/RESPECT.2018.8491718

[45] Jakita O. Thomas, Yolanda Rankin, Rachelle Minor, and Li Sun. 2017. Exploring the Difficulties African-American Middle School Girls Face Enacting Computational Algorithmic Thinking over three Years while Designing Games for Social Change. Computer Supported Cooperative Work: CSCW: An International fournal (2017). https://doi.org/10.1007/s10606-017-9292-y

[46] Alicia Nicki Washington. 2020. When Twice as Good Isn't Enough: The Case for Cultural Competence in Computing. In Proceedings of the 51st ACM Technical Symposium on Computer Science Education (Portland, OR, USA) (SIGCSE '20). Association for Computing Machinery, New York, NY, USA, 213-219. https: //doi.org/10.1145/3328778.3366792

[47] G. J. Washington, M. Meijias, and L. Burge. 2020. Understanding How to Engage Black HS Boys in Computer Science Through Tech Innovation and Entrepreneurship. Computing in Science Engineering 22, 5 (2020), 20-28.

[48] Tiffani Williams. 2020. "'Underrepresented Minority' Considered Harmful, Racist Language". https://cacm.acm.org/blogs/blog-cacm/245710-underrepresentedminority-considered-harmful-racist-language/fulltext\#comments

[49] Ryoko Yamaguchi and Jamika D Burge. 2019. Intersectionality in the Narratives of Black Women in Computing through the Education and Workforce Pipeline. fournal for Multicultural Education (2019).

[50] Stuart Zweben and Betsy Bizot. 2020. 2019 CRA Taulbee Survey. 32, 5 (2020), 3-74. 